\title{
Thermal free vibration analysis of temperature-dependent functionally graded plates using second order shear deformation
}

\begin{abstract}
This research has been conducted to approach second-order shear deformation theory (SSDT) to analysis vibration characteristics of Functionally Graded Plates (FGPôs). Material properties in FGP's were assumed to be temperature dependent and graded along the thickness using a simple power law distribution in term of the volume fractions of the constituents. FGP was subjected to a linear and nonlinear temperature rise. The energy method was chosen to derive the equilibrium equations. The solution was based on the Fourier series that satisfy the simply supported boundary condition (Navier's method). Numerical results indicated the effect of material composition, plate geometry, and temperature fields on the vibration characteristics and mode shapes. The results revealed that, the temperature field and volume fraction distribution had significant effect on the vibration of FGPs. It was observed the second order theory was very close to the other shear deformation theorem as reported in the literature.
\end{abstract}

Keyword: Free vibration; Functionally graded plate; Second order shear deformation; Temperature dependent 